\title{
Influência do campo de velocidade na correção de amplitude, empilhamento e migração
}

Jeferson B. Fonseca*, CPGG/IGEO/UFBA, Wilker E. Souza, Rafael R. Manenti, Milton J. Porsani CPGG/IGEO/UFBA \& INCT-GP/CNPq/Brasil

\begin{abstract}
In this paper, we show how the velocity field influences various stages of the seismic data processing such as amplitude correction, stacking and migration. To show this influence we performed reprocessing in a seismic line of the Parnaíba Basin. We test the velocity analysis, amplitude correction, stacking and migration. The final results is affected by the presence of the lower signal to noise ratio.
\end{abstract}

\section{Introdução}

A análise de velocidade é uma etapa fundamental do processamento CMP de dados sísmicos, pois a qualidade da seção sísmica dependerá do grau de precisão na determinação das velocidades das camadas em subsuperfície. Com isso, esta etapa do processamento interfere diretamente outras etapas, como correção de divergência esférica, empilhamento e migração.

$\mathrm{Na}$ correção de divergência se faz necessário um campo de velocidade prévio, para poder realizar a recuperação da amplitude do sinal com tempo de propagação de energia.

No empilhamento, as velocidades de empilhamento obtidas na análise de velocidade são usadas para uma correção NMO, no qual após a correção, é feita uma soma aritmética dos traços horizontalmente da família CMP. O resultado dessa soma é o traço empilhado, o qual possui uma melhor razão sinal ruído, comparado com a família CMP antes da correção (Souza, 2014a).

A migração move os refletores com mergulho para suas verdadeiras posições em subsuperfície e colapsa as difrações aumentando a resolução espacial produzindo uma imagem sísmica da subsuperfície. Essa correção de posição dos refletores depende diretamente do campo de velocidade, pois se não obtivermos a velocidade mais próxima da verdadeira na etapa de análise de velocidade, não recolocaremos o refletor em sua posição real.

\section{Análise de Velocidade}

A correção normal moveout é a base para determinar velocidades de dados sísmicos. As velocidades calculadas, a partir da correção NMO, podem ser utilizadas para corrigir as reflexões em uma família CMP antes do empilhamento. Na qual a correção é dada pela equação (1) abaixo:

$$
\Delta t_{n m o}=t(x)-t(0)=t(0)\left[\left[1+\left(\frac{x}{t(0)_{n m o}}\right)^{2}\right]^{\frac{1}{2}}-1\right]
$$

em que:

$$
t(x)^{2}=t(0)^{2}+\frac{x^{2}}{v_{n m o}^{2}}
$$

A equação (2) descreve uma reta no plano $t^{2}-x^{2}$, onde o coeficiente angular da reta é igual $1 / V_{n m o}^{2}$, onde $V_{n m o}$ é a velocidade que melhor ajusta a hipérbole de reflexão.

A partir da equação da hipérbole, vários métodos de análise de velocidades foram criados, um deles, já citado acima, é o $t(x)^{2}-x^{2}$, onde os pontos da hipérbole de reflexão são plotados no plano e o coeficiente angular da reta fornece a velocidade da hipérbole. O problema desse método é o consumo de tempo, pois necessita da leitura $t(x)$ de cada offset.

Outro método de análise de velocidade é o CVS. O CVS consiste em gerar várias seções zero-offset de velocidade constante em um determinado range de velocidade, desta forma se tem $\mathrm{n}$ painéis de velocidade constante, onde podemos visualizar como os eventos se comportam pra cada velocidade.

O método mais comumente usado é o painel de velocidade "tempo duplo de incidência normal $\times$ velocidade". O painel é gerado percorrendo os traços com várias hipérboles em uma janela de tempo, a partir do ápice da hipérbole $\left(t_{0}, n\right)$, as quais são geradas variando a velocidade em um range. As hipérbole são corrigidas por NMO e empilhadas para cada velocidades, formando picos no domínio $T(0) \times V_{r m s}$. Após o empilhamento de todo o range de velocidade é utilizado um novo ápice e se repete o processo.

Normalmente, associado ao espectro de velocidade, aplica-se uma medida de coerência (normalmente a semblance) para facilitar o reconhecimento os eventos de reflexão, evidenciando as energias correspondentes às reflexões (Souza, 2014a).

\section{Correção de Amplitude}

A correção de amplitude se faz necessário devido a vários fatores que colaboram para a atenuação da energia do sinal sísmico com a propagação da onda. Dentre esses fatores podemos destacar divergência esférica, a absorção, perdas por transmissão entre as interfaces, reflexões múltiplas, curvatura da superfície do refletor e dispersão (Abreu, 2005).

Sabe-se que quanto mais distante da fonte a frente de onda se encontra, maior a perda de amplitude (Souza, 2014b). Essa perda está associada à lei de conservação de energia, na qual define que a intensidade de energia $\rho$ é inversamente proporcional ao quadrado do raio que representa o descolamento da frente de onda ( $r$ ), assim:

$$
\rho \alpha \frac{1}{r^{2}}
$$

onde, $\rho$ é a intensidade ou densidade de energia por unidade de superfície e r é o raio da frente de onda. Como 
há proporcionalidade da amplitude $\mathrm{A}$ com a raiz quadrada da densidade de energia, tem-se que:

$$
A \alpha \sqrt{\frac{1}{r^{2}}}=\frac{1}{r}=\frac{1}{v t_{p}}
$$

Sendo $v$ a velocidade do meio e $t_{p}$ o tempo de percurso. Desta forma, nota-se que a amplitude do sinal sísmico é inversamente proporcional à distância percorrida e decai com o tempo de propagação de energia, logo, é necessária uma função de correção variável com o tempo $C(t)$, de modo que:

$$
C(t) \quad \alpha \quad r=V t_{p}
$$

\section{Empilhamento}

No caso do empilhamento, no qual é dado pela fórmula,

$$
A(t)=\frac{1}{N} \sum_{i=1}^{N} a_{i}(t)
$$

sendo, $A(t)$ - amplitude do traço empilhado no tempo $\mathrm{t} ; \mathrm{N}$ - número de traços ou cobertura da família CDP; i-índice indicador do traço; $a_{i}(t)$ - valor da amplitude do traço i no tempo $(\mathrm{t})$. As velocidades são estimadas diretamente na etapa de análise de velocidade na qual a equação hipérbole fica:

$$
t(x)^{2}=t(0)^{2}+\frac{x^{2}}{v_{s t}^{2}}
$$

\section{Migração}

A migração de dados sísmicos tem por objetivo corrigir a imagem distorcida (seção sísmica empilhada ou pseudo-seção zero-offset) que representa o campo de onda temporal registrado na superfície transformandoo em outro campo, cujos refletores geológicos estarão verdadeiramente posicionados e as difrações colapsadas (Silva, 2004).

Em sua acepção geométrica, a migração, pode ser definida como o processo que permite a localização de difratores ou, a posição que foi gerada à energia (Rosa, 2010).

Considerando um pulso unitário registrado no tempo arbitrário $t_{0} e$ na coordenada $x_{0}$, a equação correspondente é:

$$
z^{2}=v^{2} t_{0}^{2}-\left(x-x_{0}^{2}\right)
$$

ou em termos do tempo,

$$
\tau^{2}=t_{0}^{2}-\left(\frac{x-x_{0}}{v}\right)^{2}
$$

onde $z$ corresponde à profundidade, $\tau$ é o seu equivalente em tempo, x é a coordenada horizontal e $v$ é a metade da velocidade de propagação do meio.

Em meios homogêneos e isotrópicos, a migração pode ser reproduzida algebricamente como:

$$
\sin \theta=\tan \varphi=v p
$$

onde, $\theta$ é o mergulho real, $\varphi$ é o mergulho aparente, $v$ é a metade da velocidade média e $p$ a vagarosidade horizontal, dada por

$$
p=\frac{d t}{d x}=\frac{\sin \theta}{v}
$$

Para determinar o deslocamento vertical e, assim terminar o processo de migração do mesmo evento, se faz necessário definir a vagarosidade vertical que é:

$$
\frac{d t}{d z}=\frac{\cos \theta}{v}=\sqrt{\frac{1}{v^{2}}-p^{2}}
$$

onde z é a profundidade. Desta forma pode-se definir o ângulo de mergulho real, que é dado por:

$$
\cos \theta=\sqrt{1-v^{2} p^{2}}
$$

Utilizando as equações (10) e (13), pode-se facilmente estimar o deslocamento horizontal e vertical aplicados pela migração, que é representando pelas seguintes equações:

$$
\begin{gathered}
x_{M}=x_{A}-v^{2} t \frac{d t}{d x}=x_{A}-z_{A} \sin \theta \\
z_{M}=v^{2} t \frac{d t}{d z}=z_{A} \cos \theta
\end{gathered}
$$

ou, em termos da migração em tempo,

$$
\frac{d \tau_{M}}{d x}=\frac{p}{\sqrt{1-v^{2} p^{2}}}
$$

Fazendo uma breve análise das equações (13) e (15), levase a conclusão que velocidades maiores que a verdadeira implica em correção excessiva. Sendo o oposto válido para velocidades abaixo da verdadeira.

\section{Metodologia}

Foi feito o reprocessamento de parte da linha sismica regional, RL001-03 da Bacia do Parnaíba,adquirida com fonte vibroseis pela empresa Global Geophysical Services Incorporated e disponibilizados pela BP (British Petroleum) no ano de 2015 para pesquisas acadêmicas como parte do projeto PABIP. Originalmente a linha foi adquirida com 480 canais, 20 s de registro e $2 \mathrm{~ms}$ de taxa de amostragem. Porém a linha foi reduzida para 200 canais, com tempo de registro de $5 \mathrm{~s}$ e $4 \mathrm{~ms}$ de amostragem a fim de se fazer um maior detalhamento da parte mais rasa da bacia. O fluxo do processamento realizado foi: Importação do em SEG-Y; geometria; correção estática; correção de ampitude; deconvolução; filtragem. Após finalizada o préprocessamento, foi iniciada a etapa de processamento avançado com: análise de velocidade; correção NMO; empilhamento; correção estática residual e migração; O software utilizado para o processamento da linha foi o Promax SeisSpace. 


\section{Resultados}

Foi utilizado o módulo Offset Amplitude Recovery, para recuperar as amplitudes perdidas referentes à divergência esférica. No qual esse módulo se utiliza de um campo de velocidade preliminar. O Resultado obtido pela correção de amplitude é visto na Figura 2.

A análise de velocidade no SeisSpace foi realizada utilizando o módulo Velocity Analysis, onde a melhor velocidade se encontra a partir da análise de coerência. Foram feitas a análise de 123 CMP's com incremento de 15 CMP's. Vale destacar que o primeiro CMP analisado foi o de valor 10997 e o último foi 12848.

A Figura 1 exibe o campo de velocidade RMS, a partir da interpolação linear dos valores das análises de velocidade dos 123 CMP's, analisados durante a escolha da velocidade. O módulo utilizado para construir o campo foi Velocity Viewer/Point Editor*.

O empilhamento foi realizado utilizando o módulo CDP/Ensemble Stack. O mesmo foi feito aplicando as velocidades obtidas na etapa anterior. O resultado do empilhamento é exibido na Figura 3.

A migração realizada foi a Kirchhfoff em tempo pósempilhamento. O módulo do SeisSpace para a realização dessa migração é Kirchhoff Time Mig. O campo de velocidade, a abertura do operador que se relaciona com a distância máxima para qual a energia pode ser espalhada e o ângulo de migração, são os parâmetros de entrada. $O$ resultado da migração pode ser visto na Figura 4.

\section{Conclusões}

A análise de velocidade é o alicerce do processamento CMP de dados sísmicos, pois é a partir dela que vamos gerar as imagens de subsuperfície.

A correção de amplitude se faz necessária, principalmente em dados de tempos de registro muito alto, no qual em tempos maiores há uma perda significativa da amplitude do sinal. O resultado a aplicação da correção de amplitude foi satisfatória onde podemos ver reflexões entre 3 e 5 s bem mais nítidas.

A migração conseguiu recolocar os refletores, em sua real posição, principalmente na parte inicial até a parte central da seção. Porém, a partir do CMP 12051, baixa razão sinal/ruído prejudicou a qualidade final da imagem.

\section{Agradecimentos}

Os agradecimentos são para o LAGEP/CPGG/UFBA, e CNPQ e PETROBRAS pelo apoio dado ao projeto INCTGG/CNPQ/MCTI. Também agradecemos à Landmark por ceder o software SEISSPACE utilizado neste trabalho. E por fim, a BP, projeto PABIP por ceder os dados para o processamento realizado.

\section{Referências}

Abreu, A. S. 2005. Processamento de Dados Sísmicos do Golfo do México usando o SEISMIC UNIX - Trabalho de Conclusão de Curso, Universidade Federal da Bahia, Instituto de Geociências, Salvador, Brasil.

Rosa, A.L.R. 2010. Análise do Sinal Sísmico. Sociedade Brasileira de Geofísica, Rio de Janeiro, 668 p.

Silva, M.G. 2004. Processamento de Dados Sísmicos da Bacia do Tacutu. Dissertação de Mestrado. Universidade Federal da Bahia, Salvador, Brasil. 126 p.

Souza, M.S. 2014. Determinação Automática da Velocidade de Empilhamento e Obtenção da Seção Zerooffset. Trabalho de Conclusão de Curso, Universidade Federal da Bahia, Instituto de Geociências, Salvador Brasil.

Souza, W.E. 2014. Processamento CMP de Dados Sísmicos Levantados no Norte da Bacia de São Francisco Utilizando o Software SeisSpace. Trabalho de Conclusão de Curso, Universidade Federal da Bahia, Instituto de Geociências, Salvador Brasil. 


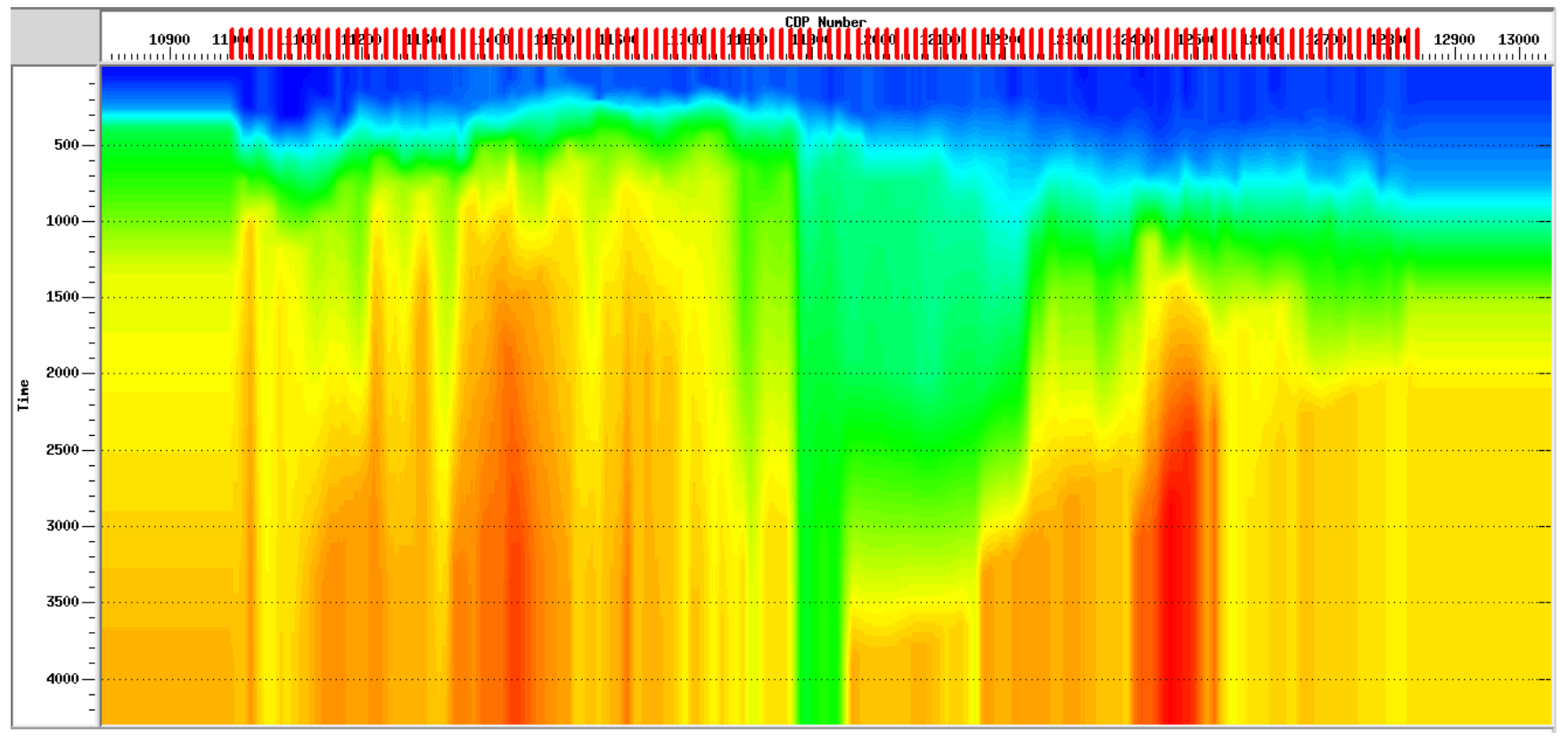

Figura 1: Campo de velocidade gerado com o módulo Velocity Viewer/Point Editor*. A partir da interpolação dos resultados da análise de velocidade.

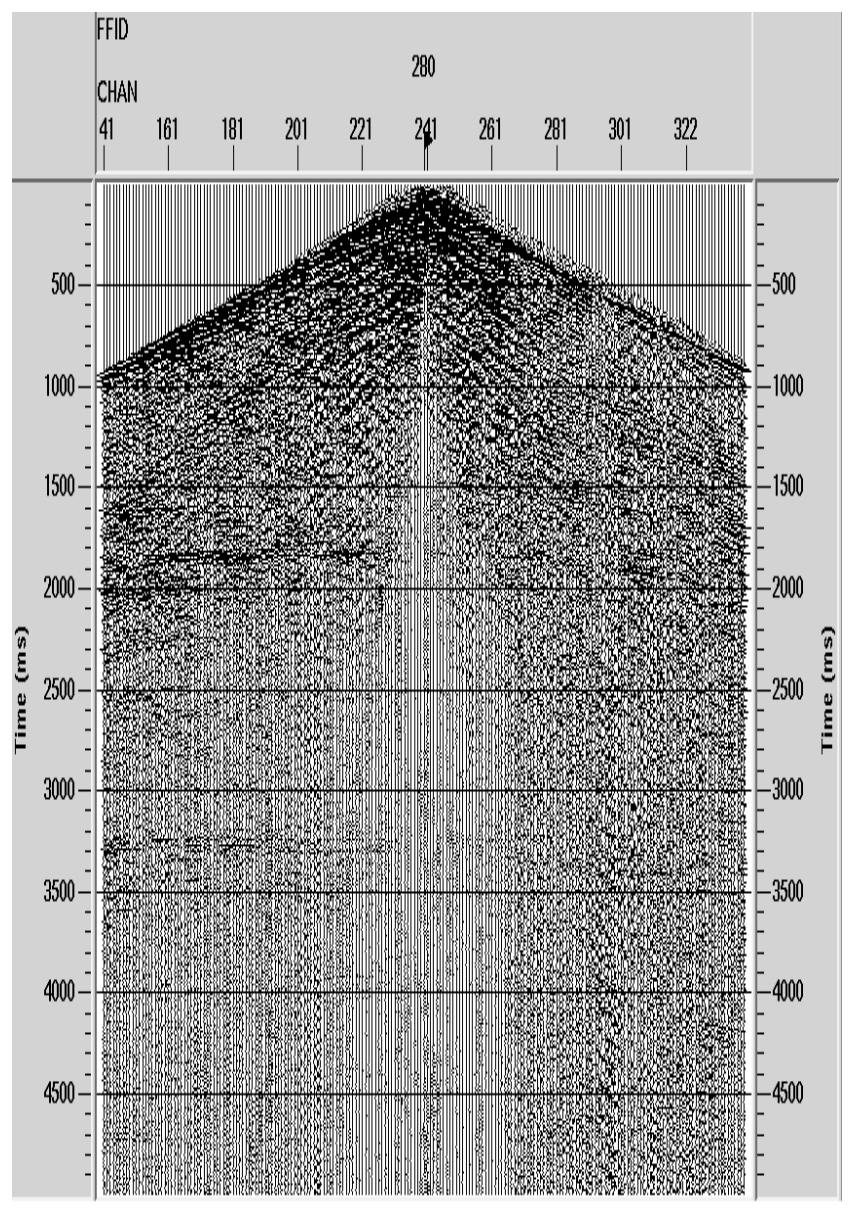

(a)

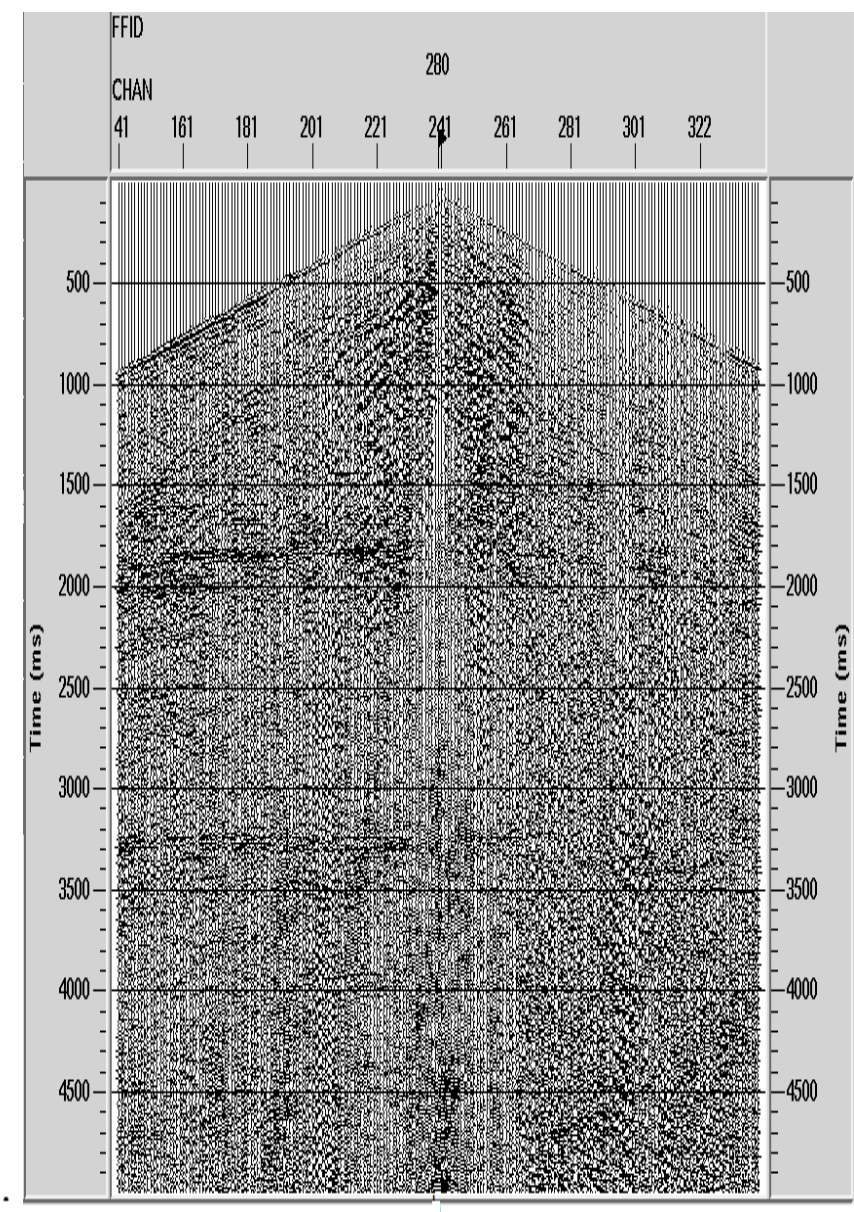

(b)

Figura 2: : Sismograma, de FFID 280, antes (a) e depois da correção de divergência esférica (b), utilizando o módulo Offset Amplitude Recovery. 


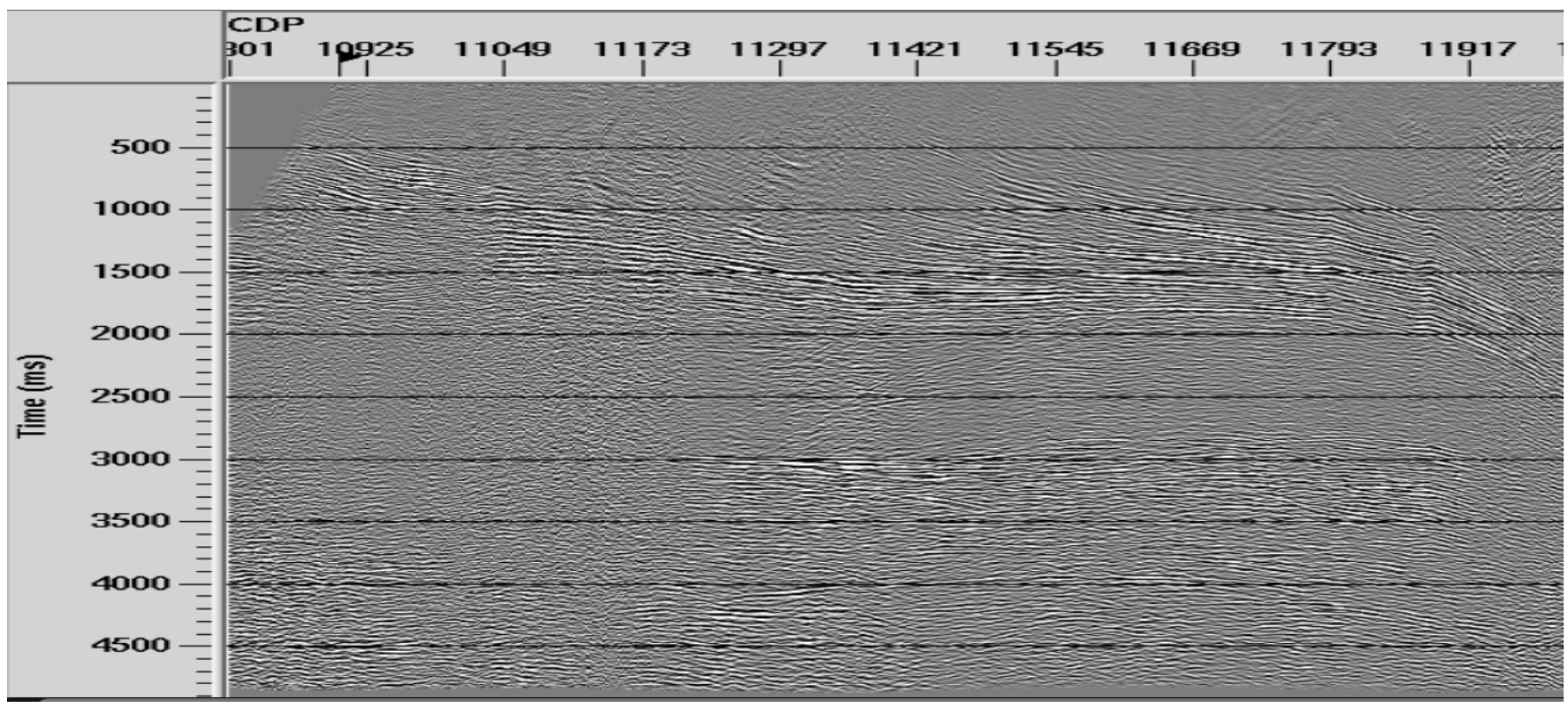

Figura 3: Seção zero-offset, gerada com o módulo CDP/Ensemble Stack, com ganho de 200\% e estática residual aplicada para melhor visualização.

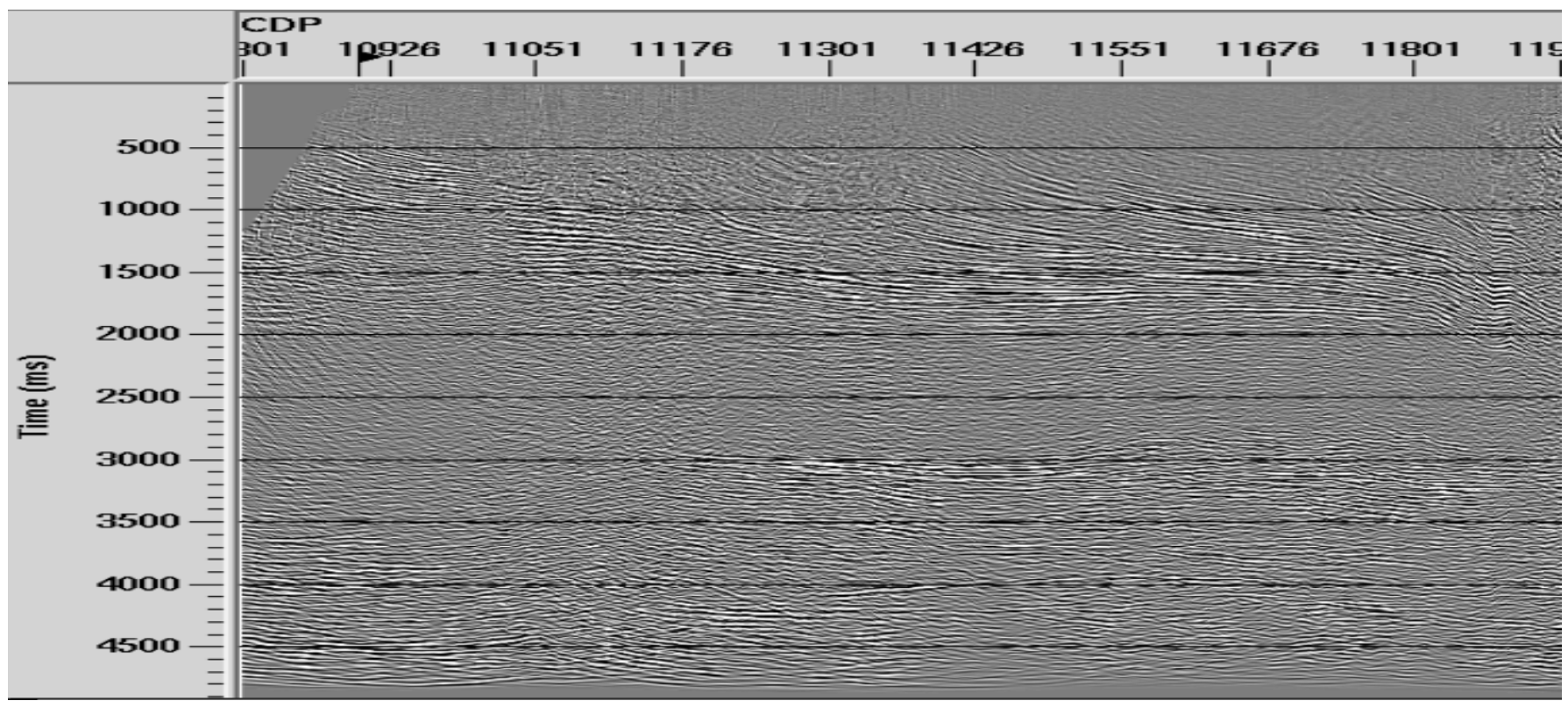

Figura 4: Seção zero-offset migrada, gerada com o módulo Kirchhoff Time Mig, com ganho de $200 \%$. 